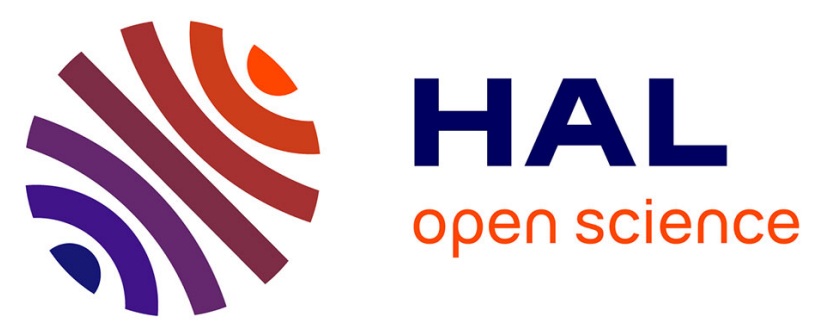

\title{
The appropriation of prosthetic devices for reading computer screens and network interactions via tactile perception
}

Loïc Deschamps, Katia Rovira, Charles Lenay

\section{- To cite this version:}

Loïc Deschamps, Katia Rovira, Charles Lenay. The appropriation of prosthetic devices for reading computer screens and network interactions via tactile perception: Analysis of patterns of use and perspective for the conception of a tactile Internet. Sciences et Technologies pour le Handicap, 2008, Technologie tactile et ses applications pour les handicaps, 2 (2), pp.159-173. 10.3166/sth.2.159-173 . hal-01609321

\section{HAL Id: hal-01609321}

https://hal-normandie-univ.archives-ouvertes.fr/hal-01609321

Submitted on 3 Oct 2017

HAL is a multi-disciplinary open access archive for the deposit and dissemination of scientific research documents, whether they are published or not. The documents may come from teaching and research institutions in France or abroad, or from public or private research centers.
L'archive ouverte pluridisciplinaire HAL, est destinée au dépôt et à la diffusion de documents scientifiques de niveau recherche, publiés ou non, émanant des établissements d'enseignement et de recherche français ou étrangers, des laboratoires publics ou privés. 


\title{
The appropriation of prosthetic devices for reading computer screens and network interactions via tactile perception
}

Analysis of patterns of use and perspective for the conception of a tactile Internet.

\author{
Loïc Deschamps ${ }^{1}$, Katia Rovira ${ }^{2}$, Charles Lenay ${ }^{1}$ \\ ${ }^{1}$ Université de Technologie de Compiègne \\ Laboratoire COSTECH - CRED \\ Centre Pierre Guillaumat \\ BP 60319 \\ 60203 Compiègne Cedex \\ charles.lenay@utc.fr \\ ${ }^{2}$ Université de Rouen \\ Laboratoire PSY.NCA \\ 76821 Mont Saint Aignan Cedex \\ katia.rovira@univ-rouen.fr
}


RÉSUMÉ. Le Système de suppléance perceptive Tactos permet la lecture tactile des formes et dispositions spatiales d'objets sur l'écran de l'ordinateur. Le projet actuel est d'étendre l'usage de ce dispositif à des situations d'interaction tactile distale via le réseau Internet. Nous rapportons ici les premiers résultats d'analyse d'usage en contexte afin de tester les possibilités matérielles et fonctionnelles entraînés par cette extension. Deux adolescents aveugles, de niveaux d'expertise différents dans l'utilisation de Tactos, ont testé différentes possibilités techniques et différents contenus. Il apparaît que l'utilisation d'effecteurs nouveaux est possible si un certain niveau d'aptitude est atteint dans l'utilisation du stylet et de la tablette graphique. De même, la question d'un recours à la bimodalité est posée afin d'améliorer le repérage dans l'espace. Pour finir, le passage à l'utilisation en "groupe" apparaît nécessaire pour la création d'un sens commun et le partage de normes.

ABSTRACT. The Tactos system of perceptual supplementation allows for tactile reading of shapes and spatial organisation of objects on computer screens. The aim of the present project is to extend the use of this device to situations of distal tactile interaction via the Internet network. Here we report the initial results of an analysis of patterns of use in context, in order to test the material and functional possibilities provided by this extension. Two blind adolescents, with different levels of expertise in using Tactos, have tested various technical possibilities and various contents. It appears that the use of new effectors is possible if a certain level of proficiency has been reached in the use of the stylus and the graphic tablet. Similarly, the question of recourse to bimodality is posed in order to improve the acquisition of reference points in space. Finally, the passage to a "group" situation seems to be necessary for the use of the device to give rise to shared meanings and the creation of norms.

MotS-CLÉS : Déficience Visuelle - Perception Tactile - Suppléance Perceptive - Design Interactif.

KEYWORDS:Visual deficiency - Tactile perception - Perceptual supplementation - Interactive design 


\section{Contexte et questions : le système TACTOS et le projet INTERTACT}

La synthèse vocale ou les barrettes de cellules brailles électroniques permettent de donner aux personnes aveugles un accès aux informations numériques. Un logiciel (ScreenReader) lit les textes présents à l'écran et donne une description des images (si les rubriques HTML sont suffisamment bien remplies). Cependant, ces systèmes ne peuvent délivrer qu'une information strictement temporelle, linéaire, suivant la succession des mots du texte ou de la description de l'écran. Celle-ci peut être plus ou moins exhaustive, mais elle ne permet pas de comprendre l'organisation spatiale de l'information: les positions des icônes et des fenêtres, les graphes et les tableaux de données, les schémas et les cartes, toutes ces informations bidimensionnelles qui font la richesse des interfaces graphiques. Outre le fait que les interfaces ordinateurs sont de plus en plus imagées, les images sont utiles pour les aveugles [Heller, 2000; 2002], ces dernières pouvant fournir des informations spatiales sous formes de cartes ou pouvant servir de support aux formalisations mathématiques.

Le système Tactos (logiciel et module tactile) a été développé pour rendre accessible aux aveugles les images et graphiques présents sur l'écran de l'ordinateur [Hanneton, Gapenne, Genouel, Lenay, Marque 1999 ; Sribunruangrit, Marque, Lenay, Gapenne, Vanhoutte 2002, 2004]. Ce système consiste essentiellement en un dispositif de commande de stimulateurs tactiles (cellule de barrette braille générant électroniquement le mouvement de petits picots) en fonction des déplacements du curseur sur l'écran d'un ordinateur. Une souris (ou un autre effecteur comme le stylet d'une tablette graphique) commande les déplacements du curseur qui correspond par exemple à une petite matrice de 16 champs récepteurs (voir figure 1). Quand un champ récepteur croise au moins un pixel noir il déclenche l'activation en tout ou rien d'un picot de la cellule braille. Le sujet est non-voyant, malvoyant ou voyant avec les yeux bandés, sa main dominante tenant l'effecteur (souris ou autre), le stimulateur tactile étant installé sous sa main libre.

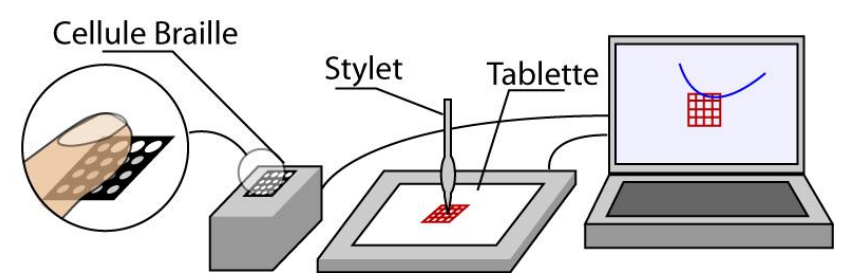

Figure 1 : le logiciel « Tactos ». Les formes inscrites dans l'espace numérique de l'écran sont perçues tactilement: leur exploration par une matrice de 16 champs récepteurs commande l'activation des 16 picots de deux cellules brailles.

Ce dispositif de suppléance perceptive permet donc l'exploration d'une image tactile virtuelle. De nombreuses observations ont permis de souligner la capacité des 
sujets à localiser, reconnaître, catégoriser et identifier des formes plus ou moins complexes [Gapenne, Rovira, Ali Ammar et Lenay, 2003; Rovira et Gapenne, 2008]. Ces objets ne sont pas donnés d'un coup au système sensoriel comme une forme qu'on appliquerait sur la peau. Il n'y a que 16 petits champs récepteurs, donc une donnée sensorielle très pauvre à chaque instant. Si les sujets réussissent à reconnaître des formes dans un espace, ce ne peut être que par leur exploration active, en intégrant dans le temps leurs mouvements et sensations tactiles. Par cette activité perceptive les sujets réalisent une forme de perception tactile des objets numériques. Mais, ce type de perception est de même nature que la perception haptique "naturelle" puisque si l'on se réfère aux travaux de Millar (1994) dans le cadre de la perception de l'espace, la compréhension des configurations spatiales s'appuie en priorité sur le codage kinesthésique des trajets manuels. Le système Tactos donne ainsi accès à l'information spatialisée : lecture et production de dessins, de graphiques, de figures géométriques, lecture de l'écran Windows et différenciation des fenêtres actives ou non, etc.

Un tel système qui permet la lecture d'écran par une sorte de toucher des formes numériques, permet aussi de «toucher » d'autres utilisateurs dès lors que l'on crée un espace numérique partagé. Pour cela, notre premier prototype consiste simplement en la mise en réseau de deux systèmes Tactos (voir figure 2). L'espace numérique partagé via le réseau permet des rencontres tactiles entre les participants aveugles ou voyants les yeux bandés.

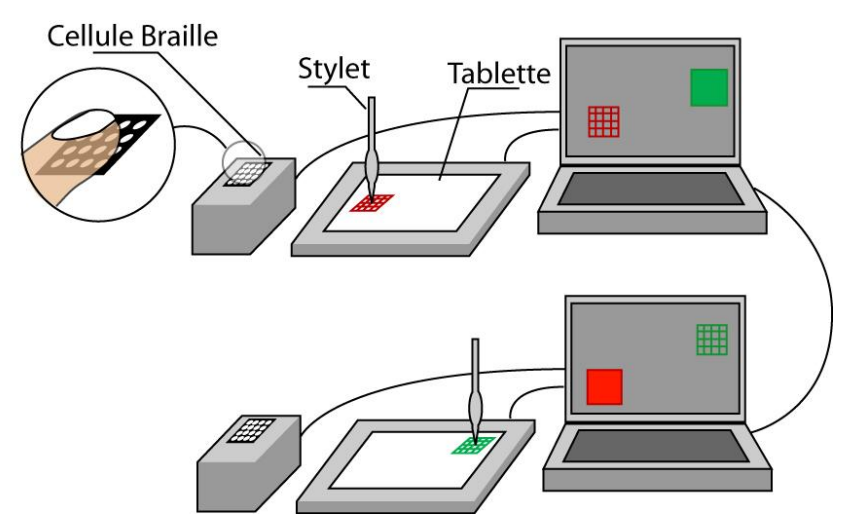

Figure 2 : Deux systèmes Tactos en réseau. Ici, les «corps-percevants » (matrices carrées de 16 champs récepteurs) et les «corps-images » (surfaces carrées de 32x32 pixels) sont identiques chez les deux partenaires.

Dans la mesure où, quand un participant «touche » l'autre, il est en même temps « touché » par lui, on peut parler d'une forme de « caresse distale ». On a donc là l'occasion d'une recherche expérimentale sur les interactions perceptives, et en 
particulier sur la perception de l'image que chacun présente à autrui au cours de cette interaction [Auvray, Lenay, Stewart, 2008]. Chaque stylet tactile commande à la fois: i) les mouvements d'une matrice de champs récepteurs couplée à autant de stimulateurs tactiles ; et ii) les déplacements d'un corps virtuel (avatar), c'est-à-dire d'un corps-image que l'autre utilisateur peut percevoir via ses propres champs récepteurs. Chacun de son coté explore sa tablette et entre en contact, soit avec des objets du milieu partagé, soit avec le corps-image (avatar) de l'autre acteur. On permet ainsi la constitution, pour chaque utilisateur, d'un espace commun où peuvent se coordonner les actions perceptives.

Notre projet actuel est donc de mettre à disposition des utilisateurs un serveur donnant accès via ce système à des interactions tactiles distales. C'est le projet du portail Intertact qui permettra le partage d'informations et l'interaction entre les utilisateurs via un «Internet tactile». Ce portail Internet correspondra à un site web tactile mettant en œuvre un ensemble de fonctionnalités nouvelles: espaces d'interaction tactile destinés en premier lieu aux personnes aveugles et malvoyantes pour l'apprentissage de la géométrie et la lecture d'écran. Notre première cible est la population des personnes ayant un déficit visuel pour l'aide à l'apprentissage de la géométrie et la lecture des informations spatialisées sur l'écran de l'ordinateur. Mais un tel serveur sera accessible de la même façon aux personnes voyantes.

Pour ces premières études d'interaction tactile nous mettons au point différents petits modules d'interaction tactile comprenant un effecteur (un touchpad) et des cellules brailles piézoélectriques (voir figure 3).

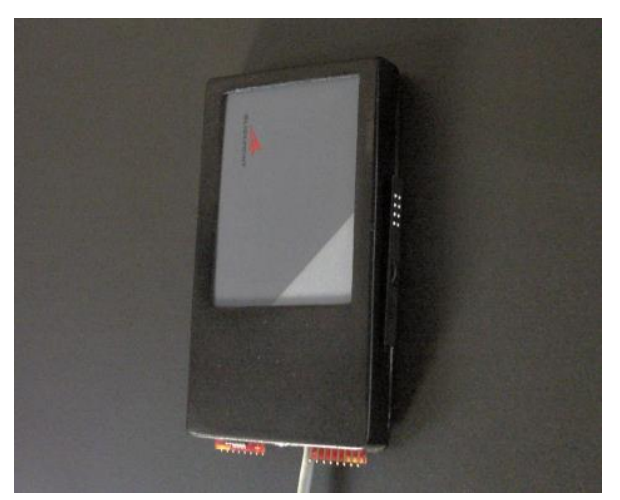

Figure 3 : Exemple de module d'interaction tactile composé d'un touchpad et de 8 picots (cellule braille piézoélectrique)

Se posent alors diverses questions tant techniques (logiciel et spécification du module d'interaction tactile) que psychologiques (la reconnaissance d'autrui, le croisement perceptif). Ceci ouvre en même temps la problématique sociologique de l'émergence d'un espace partagé au sein duquel est rendue possible la constitution de communautés d'usages (la rencontre, les mécanismes d'attention conjointe). 
Il est donc nécessaire de tester diverses possibilités matérielles et fonctionnelles entrânés par cette "extension" de l'usage de Tactos. Au niveau des modules matériels d'interaction tactile, dès les premières études l'importance de l'action a été soulignée [Bach y Rita, 1972; Lenay, Gapenne, Hanneton, Marque et Genouëlle, 2003]. Il faut donc choisir soigneusement l'effecteur le mieux adapté (Souris, Stylet, Touchpad relatif ou absolu). Au niveau des contenus à rendre disponibles sur un premier site, il faut tester quelques supports pédagogiques ou ludiques pouvant être explorés par Tactos et mis en ligne sur le site. Au niveau d'un langage d'interaction tactile, on peut commencer par tester l'usage de signes non verbaux (i.e. émoticônes tactiles) pouvant accompagner les échanges sociaux via le net.

Ce sont ces tests préliminaires que nous présentons ici.

\section{Méthodologie générale}

Afin d'envisager au mieux l'interaction tactile sur le réseau, nous avons entrepris d'étudier l'appropriation du dispositif Tactos et son utilisabilité dans des situations écologiques. Il s'agit donc de mener des analyses d'usage des «extensions» de Tactos (fonctionnalités, applications, matériel...) pour permettre le passage d'une utilisation locale à une utilisation en réseau. Le but de ces analyses d'usage est de rendre compte de l'utilisation de Tactos et de ses extensions dans une démarche évaluative, puis d'orienter la conception du dispositif Intertact en fonction des résultats obtenus et des questions soulevées.

Dans ce cadre, nous avons mis en place un suivi longitudinal de deux utilisateurs, avec deux niveaux d'expertise dans l'utilisation de Tactos (expert $=2$ ans de pratique; novice $=5$ mois de pratique). Nous privilégions une approche qualitative (mais pas uniquement) en nous centrant sur l'appropriation, l'utilisabilité et l'analyse d'usage.

Différents indices ont été retenus pour l'observation et l'évaluation des effecteurs, des supports pédagogiques et des émoticônes :

- Performances obtenues dans différentes tâches (identification, reconnaissance, estimation, localisation, navigation...)

- Temps d'exploration / de résolution de la tâche

- Nombres de pertes de l'objet à explorer entraînant une désorientation

- Stratégie d'exploration privilégiée par le sujet

- Caractère intégral ou partiel de l'exploration

- Verbalisations concomitantes et/ou entretiens d'explicitation 


\section{Effecteurs}

\subsection{Méthodologie}

Quatre effecteurs ont été testés durant les suivis longitudinaux de deux utilisateurs non-voyants. Le but de ces tests-utilisateurs était d'évaluer la pertinence de plusieurs types d'effecteur, en vue de les comparer à l'effecteur original (la tablette et le stylet) en termes d'appropriation et d'utilisabilité. Ces tests ont concerné d'une part, deux effecteurs absolus (la surface d'action proposée à l'utilisateur correspond à la surface de perception sur l'écran dans un rapport bijectif) : il s'agit du couple Tablette / Stylet, et d'un Touchpad configuré en absolu. Et d'autre part, deux effecteurs relatifs (la surface d'action correspond à une partie de l'espace de perception sur l'écran) : il s'agit d'une Souris, et d'un Touchpad configuré en relatif.

Deux tâches ont été proposées pour chaque effecteur. La première tâche est une tâche d'identification. Deux figures par effecteur sont proposées : un angle droit et une figure curviligne. Le sujet est placé sur la figure et n'a aucune contrainte de temps pour son exploration. S'il le souhaite, il peut être replacé sur la figure à sa demande. Le sujet doit indiquer de quelle figure il s'agit. Nous relevons la qualité de la réponse, le temps d'exploration, la qualité de l'exploration (exploration intégrale vs exploration partielle), la stratégie utilisée, le nombre de replacements sur la figure et les verbalisations produites.

La deuxième tâche est une tâche de localisation. Deux carrés sont placés sur l'écran. Sans aucun guidage, le sujet doit les retrouver, puis les localiser dans l'espace de l'écran. Nous relevons le nombre de stimuli trouvés, le nombre de stimuli correctement localisés, le temps d'exploration, la stratégie utilisée et les verbalisations produites.

Pour les effecteurs relatifs (Souris et Touchpad relatif), les bords de l'écran sont matérialisés par des bords de couleur produisant un son lorsque le curseur les rencontre.

\subsection{Résultats}

\subsubsection{Scores d'identification}

Concernant l'identification de deux configurations géométriques (un angle et une figure curviligne), nous pouvons noter un net écart entre les sujets (voir tableau 1). En effet, quand le sujet expert ne semble pas perturbé par le changement d'effecteur (excepté pour l'effecteur Souris qui a donné lieu à une erreur), le sujet novice présente des performances d'identification très différentes en fonction de la nature absolue ou relative du Touchpad. 


\begin{tabular}{|l|l|l|}
\hline & Expert & Novice \\
\hline Tablette & $2 / 2$ & $1 / 2$ \\
\hline Touchpad absolu & $2 / 2$ & $2 / 2$ \\
\hline Souris & $1 / 2$ & $1 / 2$ \\
\hline Touchpad relatif & $2 / 2$ & $0 / 2$ \\
\hline
\end{tabular}

Tableau 1 : Scores d'identification obtenus par les deux sujets avec les 4 effecteurs

Ainsi, quel que soit le niveau d'expertise, le Touchpad configuré en absolu offre les meilleurs résultats.

\subsubsection{Scores de désorientation}

Au début de la tâche d'identification, la main du sujet est placée sur la tablette, de façon à ce que le curseur «touche» la figure. La tâche débute lorsque nous lâchons la main du sujet et que ce dernier commence l'exploration de la forme. Si le sujet perd le contact avec la figure au cours de son exploration et qu'il a des difficultés à la retrouver seul, il a la possibilité d'être replacé dessus par l'expérimentateur. Nous appelons donc «score de désorientation» le nombre de replacements sur la figure demandés par l'utilisateur, ce qui témoigne de la difficulté à s'orienter dans l'espace de l'écran et à retrouver la figure perdue.

\begin{tabular}{|l|l|l|}
\hline & Expert & Novice \\
\hline Tablette & 0 & 0 \\
\hline Touchpad absolu & 0 & 1 \\
\hline Souris & 3 & 9 \\
\hline Touchpad relatif & 0 & 12 \\
\hline
\end{tabular}

Tableau 2 : Scores de désorientation obtenus par les deux sujets avec les 4 effecteurs

De façon générale, le sujet expert perd rarement le contact avec la forme lors de son exploration sauf avec le Touchpad relatif mais dans ce cas il parvient toujours à reprendre contact avec la figure (voir tableau 2). Seul l'effecteur Souris entraîne 3 demandes d'aide pour le replacement. Le sujet novice perd plus souvent le contact mais il parvient à retrouver la forme avec les effecteurs absolus. En revanche, il ne parvient pas à retrouver les figures avec les effecteurs relatifs puisque nous avons jusqu'à 12 demandes de replacements avec le Touchpad relatif. 


\subsubsection{Temps moyens d'exploration par objet correctement identifié}

Nous pouvons remarquer que l'identification correcte des objets explorés par les deux utilisateurs prend moins de temps avec les effecteurs absolus qu'avec les effecteurs relatifs (voir tableau 3).

\begin{tabular}{|l|l|l|}
\hline & Expert & Novice \\
\hline Tablette & 25,5 & 45 \\
\hline Touchpad absolu & 46 & 87,5 \\
\hline Souris & 40 & 195 \\
\hline Touchpad relatif & 145 & $\mathrm{X}$ \\
\hline
\end{tabular}

Tableau 3 : Temps moyens en secondes d'exploration avec les 4 effecteurs

Par ailleurs, les temps moyens d'exploration reflètent également un net écart entre les deux niveaux d'expertise : l'identification correcte des objets par le sujet expert prend moins de temps que l'identification correcte des objets par le sujet novice, et ce quel que soit l'effecteur. De plus, le sujet novice n'a pas réussit à identifier les figures avec le Touchpad relatif (d'où l'absence de donnée temporelle dans le tableau 3).

Ceci souligne deux points qui nous paraissent intéressants. D'une part, l'appropriation de Tactos nécessite le développement d'un savoir-faire particulier, qui permet une utilisation efficiente de l'outil [Ali Ammar, 2005; Stewart et Gapenne, 2004]. D'autre part, l'expertise de l'outil développée à partir d'un effecteur particulier (en l'occurrence l'effecteur Tablette) peut être transférée à d'autres effecteurs, à condition que le niveau de compétence initial soit suffisant. Ceci ne fait que confirmer des résultats précédemment obtenus auprès d'adultes voyants aveuglés devant tester différents effecteurs tels que la souris, la tablette ou le joystick [Sribunruangrit, 2004]. Dans cette étude l'ensemble des sujets étaient des "novices" face à Tactos et une très nette hiérarchie en faveur de la tablette était observée.

\subsubsection{Scores de localisation}

Lors de la deuxième tâche, le sujet doit retrouver deux objets (deux carrés pleins) placés aléatoirement sur l'écran et les localiser dans l'espace de celui-ci. Le tableau 4 présente ainsi le score de découverte de la cible ainsi que celui de sa localisation (scores entre parenthèses). 


\begin{tabular}{|l|l|l|}
\hline & Expert & Novice \\
\hline Tablette & $2(2)$ & $2(1)$ \\
\hline Touchpad absolu & $2(2)$ & $2(2)$ \\
\hline Souris & $2(2)$ & $1(0)$ \\
\hline Touchpad relatif & $1(1)$ & $1(0)$ \\
\hline
\end{tabular}

Tableau 4 : Scores de découverte et (localisation) obtenus avec les 4 effecteurs

Nous pouvons remarquer que les sujets ne se différencient pas en ce qui concerne la découverte des cibles sur l'écran (voir tableau 4). Par contre, le niveau d'expertise a une influence sur la capacité à localiser l'objet relativement au cadre de l'écran. Le sujet expert trouve et localise correctement les objets, sauf dans le cas du Touchpad relatif, qui ne paraît pas approprié pour ce type de tâche malgré les bords d'écran sonores. En revanche, le sujet novice éprouve de nettes difficultés avec la Souris et le Touchpad relatif comparativement aux effecteurs absolus, la meilleure performance étant obtenue avec le Touchpad absolu. Dans cette situation, les objets doivent être localisés en référence à un cadre de référence spatiale qui est ici le cadre de l'écran. Le référentiel spatial est soit de type égocentré, soit de type allocentré [Paillard, 1971]. Le deuxième type de référentiel est indépendant du point de vue du sujet et permet ainsi d'appréhender les relations entre objets. Nous pouvons faire l'hypothèse que le sujet novice a des difficultés à construire ce type de cadre de référence au cours de ses explorations lorsque les effecteurs sont relatifs.

D'ailleurs, au niveau des stratégies d'exploration, nous pouvons dire que celles-ci sont largement dépendantes de la configuration de l'effecteur (relatif vs absolu). Avec les effecteurs relatifs, la stratégie a principalement consisté à repérer et suivre les bords d'écran sonores, ce qui a empêché les sujets de s'aventurer trop loin de ceux-ci, et donc d'explorer la totalité de l'écran. Nous retrouvons ainsi une stratégie d'exploration mise en place par les aveugles lors de la découverte d'une pièce inconnue : la "stratégie du périmètre" qui consiste à recueillir des informations sur la forme et la taille de la pièce [Hill et Ponder, 1976] avant d'aller explorer les objets.

\subsubsection{Perspectives pour le projet Intertact}

Ce type de résultats nous paraît important pour la bonne conduite du projet Intertact. En effet, avec l'effecteur original (i.e. Tablette / Stylet), une source de difficulté a été soulevée grâce aux analyses d'usage. En effet, un utilisateur nonvoyant de naissance n'a que très rarement une expérience de la tenue d'un stylo, ce qui peut empêcher l'utilisation efficiente du dispositif par une contrainte extrinsèque. De cette façon, le Touchpad absolu nous paraît être une alternative intéressante. D'une part, cet effecteur permet des performances comparables à celles obtenues avec l'effecteur original. D'autre part, son utilisation semble plus intuitive que la 
manipulation du stylet sur la tablette, en se rapprochant davantage de la perception tactile naturelle.

Quoi qu'il en soit, ces études expérimentales en situation d'usage nous ont permis d'élaborer des spécifications quant à la conception d'un module d'interaction tactile destiné au portail Intertact. Ces spécifications nous amènent aujourd'hui à lancer le prototypage d'un module comprenant un Touchpad absolu. Lorsque ce prototypage sera finalisé, de nouvelles évaluations pourront être menées. A terme, l'utilisateur pourra lui-même choisir son effecteur, dans le but de garantir une meilleure plasticité du système.

\section{Supports pédagogiques numériques}

Tactos offrant la possibilité d'explorer des images numériques par la modalité tactile, le site web d'Intertact nous paraît approprié pour la diffusion de supports pédagogiques divers. Dans le cadre de l'appropriation du dispositif, nous proposons aux jeunes utilisateurs d'explorer des formes géométriques sélectionnées lors de précédentes études longitudinales [Ali Ammar, Gapenne, Blomme et Rovira, 2005; Ali Ammar, 2005]. Ces figures sont lues, identifiées ou reconnues avec plus ou moins de difficulté, mais avec une certaine efficience. Ces résultats d'observations nous permettent donc d'envisager la lecture via Tactos de supports variés d'information, tels que des cartes de géographie, des diagrammes, ou encore des graphiques, qui pourraient être disponibles en ligne via le portail Intertact.

Pour évaluer l'accessibilité de ce type de support via le dispositif Tactos, une carte de géographie a été testée lors des suivis longitudinaux. Il s'agit d'une carte de France relativement épurée, minimaliste, présentant uniquement les contours du territoire et sept villes. Celles-ci sont représentées par des points dans l'espace. Lorsque le curseur rencontre un de ces points, le nom de la ville est restitué via un dispositif de synthèse vocale "Jaws de la société Freedom Scientificl" pour Windows. Nous avons demandé aux sujets d'explorer librement cette carte, et d'exprimer leur satisfaction ou leurs difficultés.

Les résultats de ces tests sont de deux ordres. D'une part, nous observons que ce type de support permet un accès à l'information, les sujets ayant pu localiser quelques villes et explorer le contour de la carte. D'autre part, l'analyse d'usage et les verbalisations ont soulevé un problème de guidage au sein de l'espace. En effet, il apparaît que l'exploration se fait « au hasard », jusqu'à « rencontrer » une ville de façon fortuite, sans nécessairement permettre la recherche intentionnelle d'une information. Cela montre encore une fois que les sujets transposent à la situation Tactos les stratégies mises en place lors de l'exploration d'un espace plus large. En effet, dans le cadre de la localisation d'objets dans une pièce, certains sujets choisissent un objet comme repère pour aborder les relations spatiales entre cet objet

\footnotetext{
${ }^{1}$ www.freedomscientific.com
} 
et les autres [stratégie du "point de référence" Tellevik, 1992]. Les observations sont tout de même encourageantes et nous amènent à de nouvelles spécifications. A ce jour, un système de guidage tactile et/ou sonore est en cours d'élaboration pour permettre la recherche d'information, et sera testé également en situation d'usage. Il s'agit de faire converger des « lignes-guides » depuis le contour de la carte jusqu'à la ville recherchée, préalablement saisie dans un moteur de recherche. Ce système de guidage perceptif pourrait permettre d'explorer l'intérieur de la carte de façon systématique, à condition que cela ne gêne pas l'exploration du cadre général. L'intérêt du recours à la bimodalité (tactile + auditif) a d'ailleurs déjà été repéré dans le contexte de l'exploration de formes mathématiques [Ali Ammar, Gapenne, Lenay et Stewart, 2002].

\section{Emoticônes tactiles}

L'interaction tactile sur le réseau médiatisée par ce dispositif soulève de nombreuses questions. Parmi elles, nous nous demandons quelle valeur émotionnelle peut être attribuée à la stimulation tactile, de façon à permettre une interaction porteuse de sens entre deux ou plusieurs utilisateurs. Plus précisément, dans le cadre d'une communication asynchrone de type Messenger, la question est de savoir ce que pourrait apporter la modalité tactile à la dynamique interactionnelle.

Nous avons donc élaboré et testé quelques émoticônes tactiles, avec pour objectif de trouver une alternative aux schémas visuels utilisés dans la communication asynchrone sur Internet (les «Smileys» qui traduisent l'état émotionnel de l'émetteur). Le but est de pouvoir figurer les indices non-verbaux et paraverbaux de la communication, de façon tactile, pour enrichir les interactions verbales.

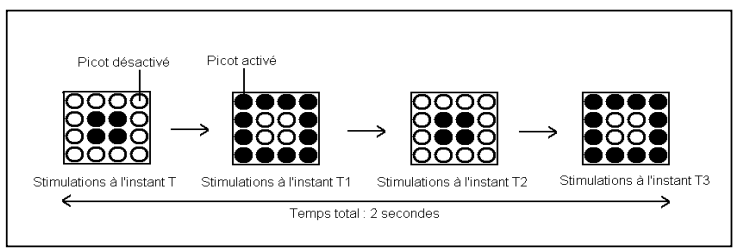

Figure 4 : Exemple de l'émoticône tactile de la joie.

La figure 4, ci-dessus, fournit l'exemple de l'émoticône tactile de la joie. L'état émotionnel de "joie" est figuré par l'alternance d'expansion et contraction du champ de stimulation. Après un premier test auprès des deux adolescents, il apparaît que ces séquences de stimulations n'ont pas de sens en dehors de l'utilisateur qui les a produites : l'émergence d'un sens est possible dans le cadre d'une convention entre n utilisateurs, mais pas dans celui d'une «norme » de communication. D'où plusieurs questions soulevées, comme par exemple celle qui concerne les conditions d'émergence d'une signification à partir d'une stimulation tactile arbitraire. Lors de 
prochains tests, nous tenterons de préciser ces conditions dans différents contextes de communication. Lors de l'analyse d'usage, les utilisateurs ont tout de même exprimé leur souhait d'avoir accès à ces émoticônes, et éventuellement de pouvoir en créer par eux-mêmes. Une telle fonction laisserait ainsi la possibilité aux utilisateurs de convenir de leur signification dans le cadre d'une interaction.

Les différentes remarques présentées par les utilisateurs ouvrent sur la question de la définition des «corps-images » (c'est-à-dire des avatars tactiles). En effet, audelà d'un simple signe interactionnel ponctuel, le fait de pouvoir élaborer sa propre configuration de stimulateurs tactiles pourrait permettre une définition personnalisée des avatars tactiles, de façon à individualiser ce que l'utilisateur laisse à percevoir pour les autres utilisateurs. De cette façon, la reconnaissance d'autrui au sein d'un espace partagé pourrait être facilitée, et enrichir le croisement perceptif.

\section{Conclusion et perspectives}

Le projet d'étendre l'usage de Tactos à une situation d'interaction en réseau nécessite tout un ensemble de tests des possibilités matérielles et fonctionnelles. Ainsi, des analyses d'usage en contexte ont permis de différencier les difficultés qui relèvent des limites du matériel, de celles liées au manque d'expertise du sujet.

Nous avons pu noter qu'un utilisateur peut changer d'effecteur (passer de la tablette au Touchpad, par exemple) à condition qu'il ait atteint un certain niveau de compétences en terme de stratégie d'exploration. De même, la localisation des objets nécessite la construction d'un référentiel allocentré, construction qui semble plus aisée chez l'expert. Le fait que notre travail s'insère dans une étude longitudinale, permet justement le développement d'expertises chez l'adolescent. De plus, il semble important que ce travail se fasse, dans un premier temps, sous la forme d'un suivi individuel afin de repérer les intérêts propres au sujet. Ceci ajouté à la collaboration avec l'équipe enseignante permet d'utiliser un matériel en adéquation avec le niveau scolaire et les intérêts du jeune.

En ce qui concerne les évolutions à apporter au dispositif, le recours à la bimodalité (visuelle et tactile) semble prometteur dans le contexte du repérage spatial. De même, il s'agit d'intégrer des techniques déjà validées auprès de voyants aveuglés afin d'améliorer l'usage de Tactos. Par exemple, la technique du zoom haptique permettrait des changements d'échelle intéressants dans le cadre de l'utilisation des cartes [Ziat, Gapenne, Lenay, Lecolinet, Mouret \& Stewart, 2006; Ziat, Gapenne, Stewart \& Lenay, 2007].

Les observations présentées ici ne sont qu'une des premières étapes dans un processus itératif de développement techniques, d'analyse des usages, de développement technique et de constitution de communauté de pratique qui est ici a son commencement et qui devrait pouvoir impliquer toutes les utilisateurs et chercheurs intéressés via un travail en réseau. 


\section{Remerciements}

Nous remercions vivement les adolescents qui participent à ce projet, ainsi que l'ensemble des professionnels du centre Normandie-Lorraine qui acceptent de nous aider dans notre travail de recherche.

\section{Références}

Ali Ammar, A., Gapenne, O., Blomme, E. \& Rovira, K., (2005), Analyse de l'exploration tactile sur support traditionnel chez la personne aveugle et conception de l'interface de lecture Tactos, Revue d'intelligence artificielle, 19 (1-2), p.339-354.

Ali Ammar, A. (2005). Analyse des explorations haptiques de formes pour la conception d'un dispositif de suppléance perceptive dédié aux personnes aveugles. Doctorat Sciences et Technologies, UTC.

Ali Ammar, A., Gapenne, O., Lenay, C. and Stewart, J. (2002). Effect of bimodality on the perception of 2D forms by means of a specific assistive technology for blind persons. In Proceedings of the Conference on Assistive Technology for Vision and Hearing Impairement (CVHI'2002), Grenade, Espagne, pp 45-52.

Auvray M., Lenay C., Stewart J. (2008) Perceptual interactions in a minimalist virtual environment, New Ideas in Psychology

Bach-y-Rita, P. (1972). Brain mechanisms in sensory substitution, Academic Press, New York.

Gapenne, O., Rovira, K., Ali Ammar, A. \& Lenay, C. (2003). TACTOS : A special computer interface for the reading and writing of 2D forms in blind people, in . C. Stephanidis (Ed), Universal Access in HCI, Inclusive Design in the Information Society, (Lawrence Erlbaum Associates, London), pp.1270-1274.

Hanneton S., Gapenne O., Genouel C., Lenay C., Marque C., (1999) Dynamics of Shape Recognition Through a Minimal Visuo-Tactile Sensory Substitution Interface, Third Int. Conf. On Cognitive and Neural Systems, Mai 1999, Boston, p. 26-29.

Heller, M.A. (2000). Touch, representation and blindness. UK: Oxford University Press.

Heller, M.A. (2002). Tactile picture perception in sighted and blind people. Behavioural Brain Research, 135, 65-68.

Hill, E. \& Ponder, P. (1976). Orientation and mobility techniques: A guide for the practitioner. American Foundation for the Blind.

Lenay, C., Gapenne, O., Hanneton, S., Marque, C. \& Genouëlle, C. (2003). Sensory Substitution, Limits and Perspectives", in Y. Hatwell and al. (Eds), Touch for Knowing, (John Benjamins, Amsterdam, 2003), pp.275-292.

Millar, S. (1994). Understanding and representing space: Theory and evidence from studies with blind and sighted children. Oxford: University Press.

Paillard, J. (1971). Les déterminants moteurs de l'organisation de l'espace, Cahiers de Psychologie, 14, 261-316.

Rovira, K., \& Gapenne, O. (2008). Catégoriser à l'aide d'un système de suppléance perceptive : stratégies d'exploration et de classification d'adolescents aveugles. In E. Loarer, P. Vrignaud, J.L. Mogenet, F. Cuisinier, H. Gottesdiener \& P. Mallet (Eds.), Perspectives différentielles en psychologie, Presses Universitaire de Rennes, pp. 399-402.

Sribunruangrit, N. (2004 Sribunruangrit N., Marque C., Lenay C., Gapenne O. and Vanhoutte C. (2002) Braille Box: Analysis of the Parallelism Concept to Access 
Graphic Information for Blind People, EMBS-BMES 2002, The Second Joint Meeting of the IEEE Engineering in Medicine and Biology Society and the Biomedical Engineering Society USA (Houston, Texas): 23-26 octobre 2002

Sribunruangrit, N., Marque, C., Lenay, C., Gapenne, O. and Vanhoutte, C., "Speedaccuracy tradeoff during performance of a tracking task without visual feedback", IEEE transactions on Neural Systems and Rehabilitation Engineering, vol. 12, no.1, (2004), 131-139.

Stewart, J. \& Gapenne, O. (2004). Reciprocal modelling of active perception of 2-D forms in a simple tactile-vision substitution system. Minds and Machines, 14, 309-330.

Tellevik, J.M. (1992). Influence of spatial exploration patterns on cognitive mapping by blindfolded sighted persons, Journal of Visual Impairment and Blindness, 86, 221224.

Ziat M., Gapenne O. Lenay C., Lecolinet E., Mouret G. \& Stewart J. (2006), Espace de perception et seuils de confort pour un zoom haptique en 2D. Revue d'Interaction Homme Machine, 7, 29-54.

Ziat M., Gapenne O., Stewart J. \& Lenay C. (2007), Haptic recognition of shapes at different scales: A comparison of two methods of interaction. Interacting with Computers, 19, 121-132. 